\title{
Multifractal analysis of low-latitude geomagnetic fluctuations
}

\author{
M. J. A. Bolzan ${ }^{1}$, R. R. Rosa ${ }^{2}$, and Y. Sahai ${ }^{1}$ \\ ${ }^{1}$ Laboratório de Física Atmosférica e Solar, Instituto de Pesquisa e Desenvolvimento, Universidade do Vale do Paraíba, \\ UNIVAP, São José dos Campos, Brazil \\ ${ }^{2}$ Laboratório Associado de Computação e Matemática Aplicada, Instituto Nacional de Pesquisas Espaciais, INPE, São José \\ dos Campos, Brazil
}

Received: 19 June 2008 - Revised: 10 December 2008 - Accepted: 13 January 2009 - Published: 5 February 2009

\begin{abstract}
The technique of large deviation multifractal spectrum has shown that the high-latitude $\left(77.5^{\circ} \mathrm{N}, 69.2^{\circ} \mathrm{W}\right)$ geomagnetic fluctuations can be described from direct dissipation process or loading-unloading regimes of the solar windmagnetosphere coupling. In this paper, we analyze the $\mathrm{H}-$ component of low-latitude $\left(22.4^{\circ} \mathrm{S}, 43.6^{\circ} \mathrm{W}\right)$ geomagnetic field variability observed during the month of July 2000 at the Geomagnetic Observatory, Vassouras, RJ, Brazil. The variability pattern during this period is a mixture of quiet and disturbed days including the Bastille Day intense geomagnetic storm on 15 July. Due to the complexity of this data, we pursue a detailed analysis of the geomagnetic fluctuations in different time scales including a multifractal approach using the singular power spectrum deviations obtained from the wavelet transform modulus maxima (WTMM). The results suggest, as observed from high-latitude data, the occurrence of low-latitude multifractal processes driving the intermittent coupling between the solar wind-magnetosphere and geomagnetic field variations. On finer scales possible physical mechanisms in the context of nonlinear magnetosphere response are discussed.
\end{abstract}

Keywords. Magnetospheric physics (MHD waves and instabilities; Plasma waves and instabilities) - Space plasma physics (Nonlinear phenomena)

\section{Introduction}

Geomagnetic time series are often generated by complex spatio-temporal dynamics of which nonlinearity and scaling are the most important processes. As supported by theoret-

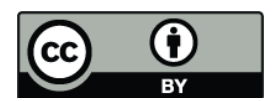

Correspondence to: M. J. A. Bolzan (bolzan@univap.br) ical and experimental studies it is known that the main geomagnetic field variation originates inside the Earth and the geomagnetic field short-term fluctuations are due to external sources. Moreover, from several high resolution observations it is possible to conclude that while the solar daily variation is approximately a regular process there is an irregular fluctuation (the geomagnetic disturbances component) caused by the solar wind-magnetosphere coupling, which is a remarkable nonlinear process. Thus, from the point of view of space weather, the complete analysis of irregular and intense geomagnetic fluctuations is relevant due to possible solar-geomagnetic coupling adverse effects on power lines and data transmission by satellites. In fact, the systematic study of the statistical properties of nonlinear magnetosphere variability and their relations to the solar activity, as the solar wind, has attracted a growing interest (e.g. Chapman et al., 1998; Chang, 1999; Kovács et al., 2001; Lui, 2002; Vörös et al., 2002; Balasis et al., 2006).

In this framework, analysis and interpretation of the geomagnetic plasma fluctuations is performed in order to characterize the solar wind influence, in terms of an intermittent energy injection, on the magnetosphere. As known from the turbulence theory the intermittency leads to deviation from usual Kolmogorov velocity structure functions and its main signature are the singularity spectra exponents, $f(\alpha)$, which represent a power-law scaling-free dependence (Frisch, 1995). For solar wind turbulence, the so-called multifractal p-model describes how solar wind energy can be distributed among scales following a multiplicative rescaling structure (Halsey et al., 1986; Meneveau and Sreenivasan, 1987). Recently, high-latitude geomagnetic fluctuations $\left(77.5^{\circ} \mathrm{N}, 69.2^{\circ} \mathrm{W}\right)$ have been analyzed using the large deviation multifractal spectrum resulting deviations from the multiplicative cascade p-model (Vörös, 2000). Actually, the multifractal analysis, in contrast to the traditional power spectrum analysis, have shown that the Hölder exponents, for

Published by Copernicus Publications on behalf of the European Geosciences Union. 

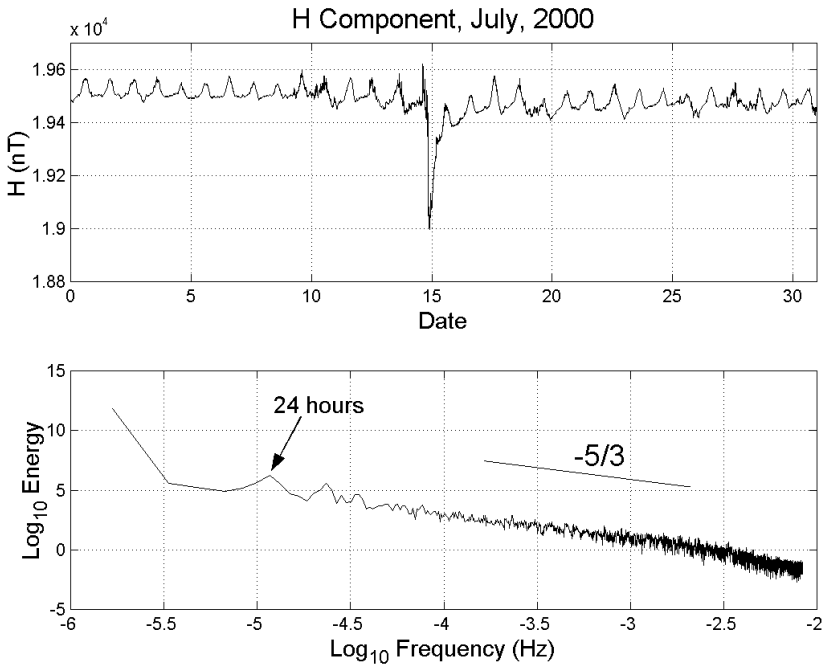

Fig. 1. Upper panel shows the $H$-component of geomagnetic field variations observed at Vassouras, Brazil, during July 2000; lower panel shows the power spectrum density of the geomagnetic field (lower curve). The straight line in the lower panel indicates the $-5 / 3$ slope.

local singularity, are time dependent showing that the flux energy at a given scale is not homogeneously distributed in time as in the traditional homogenous $1 / f^{-1}$ turbulent spectrum. A usual hypothesis is that the intermittency behavior is associated with the multifractal turbulence model (Muzy et al., 1991). This fact suggests that the fluctuations can be described by means of a multifractal scaling law which is associated with intermittency, then admitting that nonlinear and coherent processes can coexist (Vörös et al., 2002; Weygand et al., 2005; Wanliss et al., 2005). Thus, the present study deals with the multifractal analyses of low-latitude geomagnetic $H$-field records which is fully intermittent. In this sense, the continuous wavelet transform and its maxima can be used to reveal the underlying structure of the $\mathrm{H}$ component time series and its correspondent relevant time scales.

Despite the simplicity of our approach based on a single data set, at least three relevant aspects are addressed in this paper: (i) the comparative study for low-latitude considering the multifractality and characteristic time scales found for high-latitude geomagnetic fluctuations, (ii) the need of physical interpretation in the context of low-latitude; and (iii) the effectiveness of the geomagnetic fluctuations analysis based on the Wavelet Transform Modulus Maxima (WTMM).

\section{Data and methodology}

Nonlinear geomagnetic field fluctuations, in high and low latitudes, have been observed by several geomagnetic observatories. Figure 1 shows the geomagnetic fluctuations corresponding to the 1-min time resolution $H$-component time
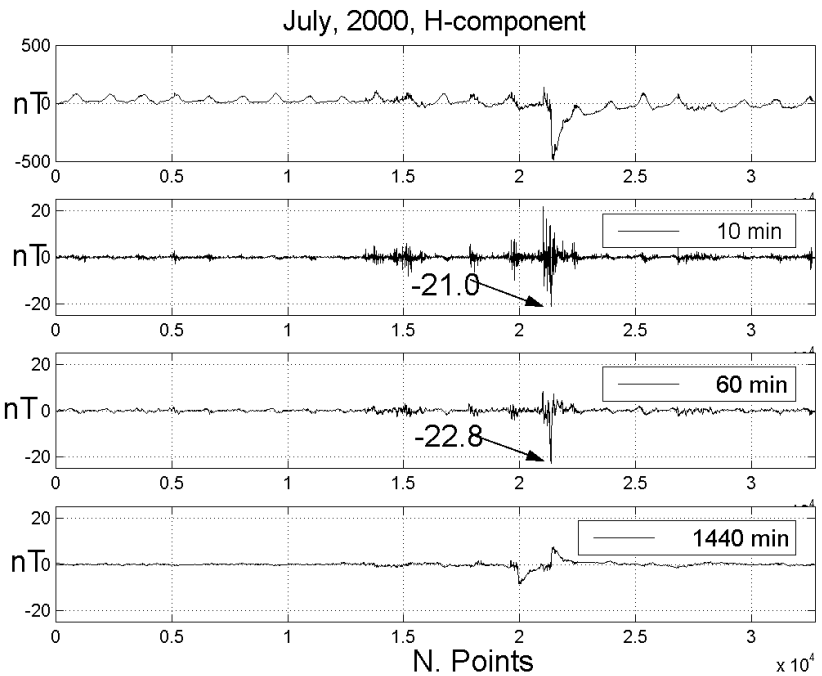

Fig. 2. First above panel is the original time series; the three below panels represent the decomposition results by three time scales displayed on legend.

series observed during the month of July 2000 at Vassouras Geomagnetic Observatory $\left(22.4^{\circ} \mathrm{S}, 43.6^{\circ} \mathrm{W}\right)$ in Brazil $(\mathrm{Pa}-$ pas et al., 2006). Its variability pattern presents a characteristic scenario on the evolution of the geomagnetic field, notably the rapid change of the field from a quiescent (quiet) period ( 1 to 9 days) to a highly fluctuating regime. Thus, the analysis has been carried out in two parts of the time series: the full data consisting of 44640 measurements and 12960 data points corresponding to the first nine days.

In order to study low latitude magnetic storm the time series showed in Fig. 1 has been previously analyzed due to the presence of a very intense geomagnetic disturbance with $D_{s t \text { max }}<-200 \mathrm{nT}$ (Bolzan et al., 2005a). However, for a detailed analysis the full $\mathrm{H}$-component record can be interpreted as composed by, at least, four main phases: (i) a quasi-regular variability before the intense disturbance, (ii) an impulsive phase, (iii) the gradual relaxation of the erupted disturbance, and (iv) the recovery phase to the nonlinear oscillating regime. It is important to mention that, following the usual criteria the disturbed period, as shown in Fig. 2, has been characterized by $D_{s t}<-50 \mathrm{nT}$ (Wanllis et al., 2005).

In the present context, as suggested by Kovács et al. (2001), neglecting the detailed reason for the evolution of the geomagnetic disturbances (storms or substorms) the fluctuating nature of the field can be interpreted, in a general framework, as the manifestation of turbulent phenomena that take place within the complex magnetosphere MHD configuration. Usually, turbulence is interpreted by means of structure functions following power-laws in the inertial range of the energy spectra. The power spectral index for the homogenous fluid turbulence is $-5 / 3$ (Kolmogorov, 1941) obtained through standard theoretical considerations, while an example determined from simulated MHD turbulence is $-3 / 2$ 
(Kraichnan, 1965; Biskamp and Schwarz, 2001). Recent studies on scaling and singularity characteristics of geomagnetic fluctuations into the MHD flow system, considering solar wind and ionosphere phenomena, suggest that the nearEarth magnetosphere is a non-homogenous interconnecting multi-scale environment where spatio-temporal variability is intermittent. In this inhomogeneous scenario, multifractal turbulence can coexist with coherent global processes and self-organized criticality involving the singular behavior of the energy distribution in physical space resulting in strong gradients (Vörös et al., 2002). Such strong variations constitute the intermittency pattern in the time-series, usually a measurement of the energy related to the physical quantities of the system, e.g. velocity (Ramos et al., 2004), temperature (Bolzan et al., 2002) or magnetic fields (Kovács et al., 2001; Lui, 2002). Thus, an appropriate characterization of the geomagnetic fluctuations is insured based on the so-called singularity spectra, $f(\alpha)$, which quantifies the deviations of the observed singularities $\alpha$ from the expected value and gives a measurement of the intermittency level (Vörös, 2000). In fact, the underlying time scales involved in the intermittent level and their different types of $f(\alpha)$ spectra can characterize the respective underlying physical mechanism, for example, involving the magnetosphere direct multi-scaling dissipation or intermittent magnetosphere response due to the solar wind nonlinear influence.

Based on the arguments discussed before, we firstly calculated the power spectral density (PSD) for the $H$-component time-series of July 2000. The least square fitting for PSD inertial range, $-(1.96 \pm 0.32)$, is shown in the lower panel of Fig. 1. The subrange scale ends are $1.65 \times 10^{-4} \mathrm{~Hz}$ and $2.10 \times 10^{-3} \mathrm{~Hz}$, approximately. Although this value, considering its inferior standard deviation, is fairly close to $-5 / 3$, its deviation from the Kolmogorov inertial range can be possibly a phenomenological signature of intermittence. This result makes evident the importance of studying, in this frequency range, the presence of intermittent phenomena driving the geomagnetic fluctuations.

Although multifractal scaling, associated with multiplicative cascades, provides a quantitative interpretation of a wide range of physical heterogeneous processes, there are few robust mathematical methods for multifractal analysis of intermittent patterns (e.g. Bunde and Havlin, 1996; Struzik, 2000). Here, we have considered, in order to obtain the singularity spectrum $f(\alpha)$ from the $H$-component time series, the Wavelet Transform Modulus Maxima (WTMM) (Muzy et al., 1991; Mallat and Hwang, 1992). The basic idea behind the WTMM method is to describe a partition function over only the modulus maxima of the wavelet transform of a signal $H(t)$.

The wavelet transform of $H(t)$ is written as (Eq. 1, p. 64 from Enescu et al., 2006)
$W_{\psi}[H](s, b)=\frac{1}{\sqrt{s}} \int_{-\infty}^{\infty} H(t) \psi^{*}\left[\frac{t-b}{s}\right] d t, \quad s>0$

where $s, b$ are real, $s>0$ and $\psi^{*}$ is the complex conjugate of a continuous wavelet function $\psi$. This transformation gives the coefficient of the wavelet decomposition of the signal $H(t)$ at time $t=b$ for scale $s$ (e.g. Enescu et al., 2006). For analysis where the variability pattern contain nonstationary power at many different scales, such as $H(t)$, a wavelet analysis based on a plane wave modulated by a Gaussian is required. Thus, it is then considered the Morlet wavelet, here taken in its form to satisfy the so-called admissibility condition (Farge, 1992; Weng and Lau, 1994):

$\psi(t)=\pi^{-1 / 4} e^{i \omega t} e^{-t^{2} / 2}$,

where $\omega \geq 5$.

The scaling and translation of this mother wavelet function over the signal $\mathrm{H}(\mathrm{t})$ are performed by the parameters $s$ and $b$. While the scale parameter $s$ stretches (or compresses) the mother wavelet to the required resolution, the translation parameter $b$ shifts the basis functions to the desired time location.

It can be shown that the wavelet transform can reveal the local characteristics of $H(t)$ at a point $t_{0}$. More precisely, we have the following power-law relation (Eq. 4, p. 64 from Enescu et al., 2006):

$W_{\psi}[H]\left(s, t_{0}\right) \approx|s|^{\alpha\left(t_{0}\right)}$

where $\alpha\left(t_{0}\right)$ is the Hölder exponent (or "singularity strength"). Thus, the exponent $\alpha\left(t_{0}\right)$, for fixed location $t_{0}$, can be obtained from a log-log plot of the wavelet transform amplitude versus the scale $s$. However, this power-law characterization is difficult when the process is governed by a hierarchical distribution of singularities compromising the exact determination of $\alpha$ on a finite range of scales. In such case any transformation of the signal $H(t)$ may obey some renormalization operation involving multiplicative cascades and it has been demonstrated that the local maxima of $\mid W_{\psi}$ $(s, b) \mid$ at a given scale $s$, are likely to contain all the hierarchical distribution of singularities in the signal. At a given scale $s$ each one of the WTMM bifurcates into new two maxima giving rise to a rich multiplicative cascade in the limit $s \rightarrow 0$. Thus, it is possible to identify a space-scale partitioning over the maxima distribution and, consequently, a usual "thermodynamical" method of computing the multifractal spectrum of $H(t)$ is to define a partition function which scales, in the limit $s \rightarrow 0$, as (Eqs. 5 and 6, p. 65 from Enescu et al., 2006):

$Z(s, q)=\sum_{n}\left|W_{\psi} H\left(s, t_{n}(s)\right)\right|^{q} \approx s^{\tau(q)}$,

where $t_{n}$ is the position of all local maxima at a fixed scale $s$ and $q$ is the moment of the measure distributed on the WTMM hierarchy, used to define the power-law scaling of 

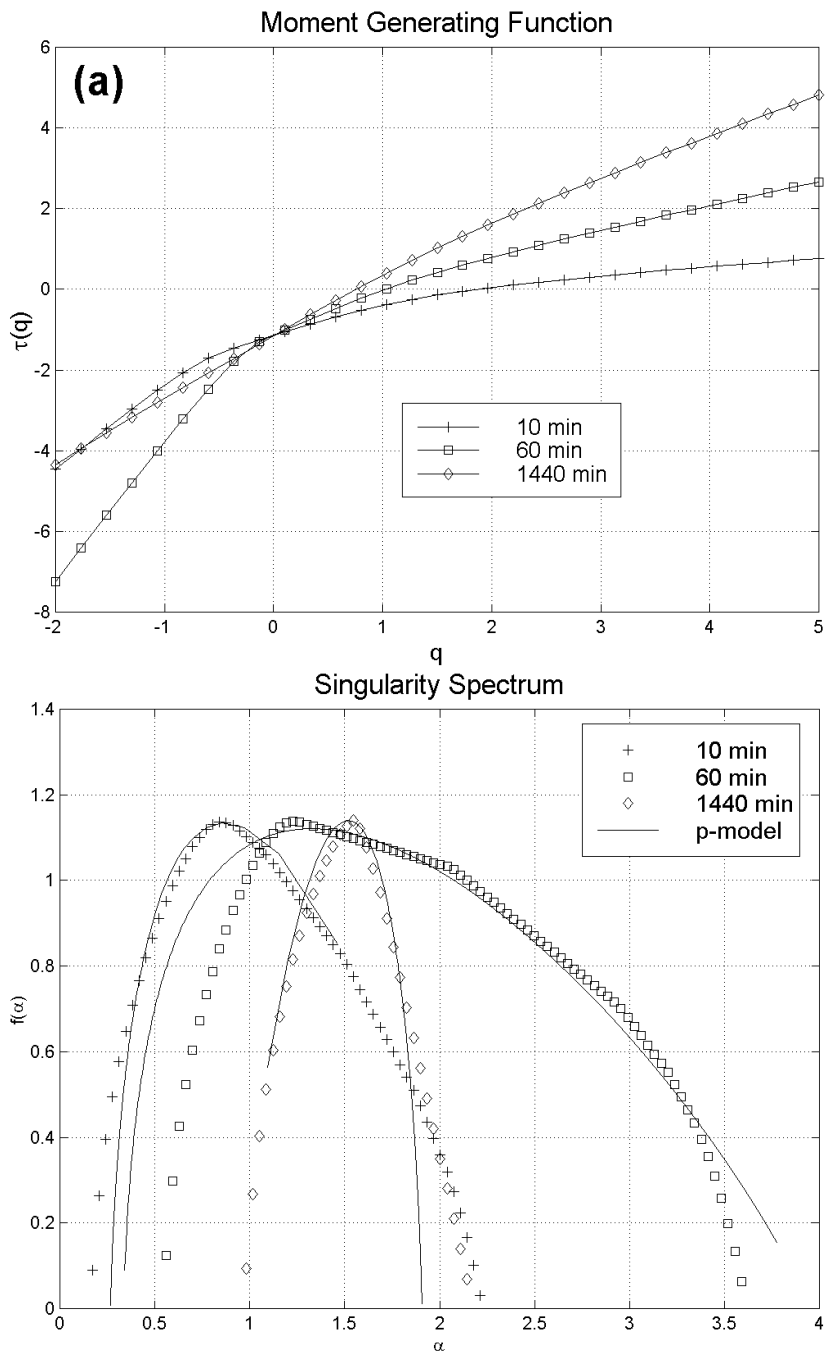

Fig. 3. (a) Tau-spectra for three time scales for July 2000. (b) Singularity spectra for H-component geomagnetic field for the same time scales from Fig. 2 and p-model (solid lines).

$Z(s, q)$. This power-law yields for small $s$ the scaling exponents $\tau(q)$ - the multifractal spectrum. Actually, there is hierarchy of the WTMM that has been used for defining the partition function $Z(s, q)$ based on the multifractal formalism (Arneodo et al., 1995).

The final step in the WTMM method used here is to examine, for a set of scales $s$, the correspondent singularity spectrum $f(\alpha)$. If one finds a single value $\alpha$ for all singularities $t_{n}$, the signal has a monofractal structure. However, if the underlying process is multifractal, then different parts of the signal are characterized by different values of $\alpha$ (Oswiecimka et al., 2006). As usual, in our approach the singularity spectrum $f(\alpha)$ can be interpreted as the negative of the Legendre transform of the multifractal spectrum $\tau(q)$, so that $f(\alpha)=-(\tau(q)-q \alpha)$ (Vicsek, 1993). Thus, from the renormal-
Table 1. Derived $\alpha$ exponents for tree characteristic time scales.

\begin{tabular}{cc}
\hline Scale (min) & Range of $\alpha$ \\
\hline 10 & $0.20-2.20$ \\
60 & $0.55-3.55$ \\
1440 & Narrow width \\
\hline
\end{tabular}

ization theory in statistical mechanics, interpreting the values of $\alpha$ as an energy cascade, the Legendre transform $f(\alpha)$ is thus the analogue of the informational entropy (Stanley and Meakin, 1988).

\section{Data analysis}

Once the Morlet wavelet function is chosen, it is necessary to choose a set of characteristic time scales in the $H(t)$ time series to use in the wavelet transform. Upholding the daily variability component, we use the concept of scale $(r)$ through the difference $\Delta H(t, r)=H(t+r)-H(t)$. As a representative set of physical oscillations in Fig. 1, we choose three characteristics scales: 1 day, $1 \mathrm{~h}$ and $10 \mathrm{~min}$. Thus, the respective signal components for scales $r$ (1440, 60 and $10 \mathrm{~min}$ ) are shown in Fig. 2. Due to the time series length reduction imposed by $\Delta H(t, r)=H(t+r)-H(t)$, the number of points in the time series of Fig. 2 was reduced to 32768 . This procedure is necessary in order to have the time series and their decompositions with the same length. Note that, this is the reason why we choose to show the time series domain, in Fig. 2, as the number of points representing the time series length.

Using Eq. (4), the WTMM is applied on the components of $H(t)$ showed in Fig. 2. When the $\tau(q)$-spectrum is approximately constant for all $q$ values, the variability pattern of the signal is classified as monofractal, otherwise, the it is classified as multifractal. Figure 3a shows the $\tau(q)$-spectra for time series shown in Fig. 2, having deviations of order $10^{-2}$ so small they are not visible in the spectra. Note that the spectrum is curved for 10 and $60 \mathrm{~min}$ time scales, and slightly curved for 1440 min scale. Complementarily, the correspondent dependence of $f(\alpha)$ on $\alpha$ is given in Fig. 3b. For comparison, we also show the singularity spectrum obtained using the p-model. We use an algorithm to fit the experimental and theoretical data, where the parameters values from p-model $\left(p_{1}, p_{2}, l_{1}\right.$ and $\left.l_{2}\right)$ are presented in Appendix A. Note that the $f(\alpha)$ spectra for $H(t)$ are of non-parabolic shape, deviating from the p-model (the continuous curve). Actually, there is a good agreement between singularity spectra for p-model and the extreme scales of 10 and $1440 \mathrm{~min}$, while the $f(\alpha)$ for $60 \mathrm{~min}$ time scale is quite different. In fact, the $60 \mathrm{~min}$ time scale is associated with strong phenomena on geomagnetic system, leading it in a phase transition. 

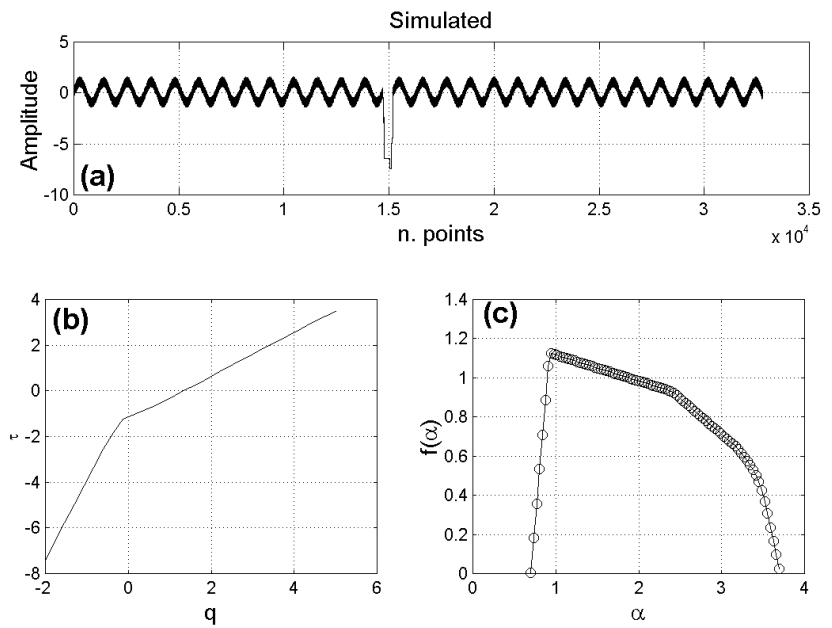

Fig. 4. (a) Simulated time series and its correspondent $\tau(q)$-spectra (b) and $f(\alpha)$ spectra (c).

According to Vörös (2000), the p-model does not allow multifractal phase transition. Furthermore, these deviations from a multiplicative process could be partially explained by sums of multiplicative measures (Vörös, 2000).

Thus, as it is expected, for 1-day period scale, there exists a well-defined low level intermittent physical oscillation, such as the diurnal and nocturnal variations (Bolzan et al., 2005a). However, for finer scales, 10 and $60 \mathrm{~min}$, nonlinear fluctuations are possibly due to the presence of multifractal processes. Moreover, using the standard interpretation for an infinite number of different exponents related to multifractal processes, the results obtained from the WTMM approach present a wide range of exponents listed in Table 1.

Thus, the finer time scales in Table 1 show a characteristic multifractal behavior. From this result, it is possible to suggest that, for shorter time scales, the energy from storm disturbance breakdowns follows a cascade process. The smaller values of $\alpha$ correspond to the burst of events, while higher values of $\alpha$ correspond to events occurring sparsely (Vörös, 2000). In this sense, the $10 \mathrm{~min}$ time scale has smaller values of $\alpha$ in comparison with other scales, indicating strong presence of fast and energetic events driving the geomagnetic field variations observed in July 2000.

It is important to note the presence of two flattening regions for $f(\alpha)$ spectra of $60 \mathrm{~min}$ scale. To study this particular spectral behavior, we generate an equivalent proxy sinusoidal time series, showed in Fig. 4a, composed by a daily periodicity summed with a white noise fluctuation. To represent the strong disturbed period an artificial breakdown, with an equivalent duration around $60 \mathrm{~min}$, was introduced in the middle region of the signal. The respective multifractal and singularity spectra are shown in Fig. $4 \mathrm{~b}$ and c, respectively. It is clear that the presence of breakdown feature in the time series cause the kink pattern on $\tau(q)$ spectrum and the double flattening in the $f(\alpha)$, otherwise it should be linear. In partic-
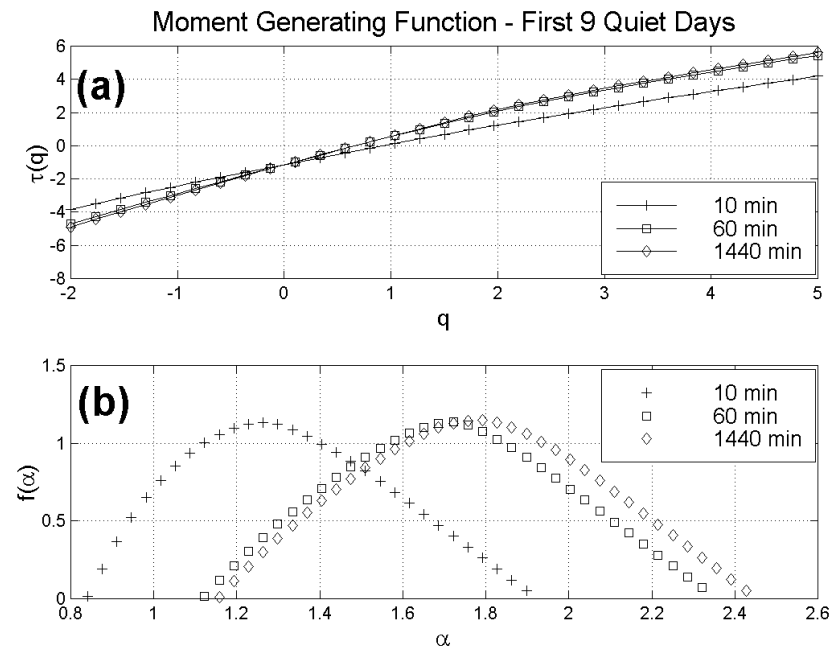

Fig. 5. (a) Multifractal spectra and (b) Singularity spectra, considering the previous three characteristic time scales, for the quasi-quite period containing the first nine days in July 2000.

ular, some structural characteristic of the $D_{s t}$ storm, mainly its duration, can be related to its signature in both the $60 \mathrm{~min}$ multifractal and singularity spectra. Thus, this result is an important finding in the present work and requires further detailed investigation.

As a consequence of the previous result, it is interesting to study the $\tau(q)$ and $f(\alpha)$ spectra taking into account the partial signal disregarding its impulsive and the after phases. Then, in order to verify the robustness of the multifractality found for the whole $H(t)$ time series, we consider, for an equivalent analysis, the partial data taking into account only the first nine days of the event. The results are shown in Fig. 5.

It should be noted that for the partial first nine days-period the absence of geomagnetic disturbances causes the linear behavior for all characteristic time scales. Although this partial time series represents a quasi-quiet period, the multifractal behavior also is well characterized in the singularity spectra (Fig. 5b). This fact may be better understood interpreting the geomagnetic field as a system out of equilibrium, comprising long-range effects from many different scales and processes. For example, according to our results, it is possible conjecture that the multifractal degree found also during the quasi-quiet period (nine days) may be due to some internal forcing related to the magnetosphere system, corroborating the results from Balasis et al. (2006).

\section{Concluding remarks}

Here, the multifractal signature for low-latitude component of geomagnetic fluctuations was successfully detected by using the Wavelet Transform Modulus Maxima and these results are in agreement with multifractal process found 
Table A1. p-model parameters obtained by maximum likelihood algorithm.

\begin{tabular}{lccc}
\hline Increment $(r)$ & $p_{1}$ & $p_{2}$ & $l_{1}$ \\
\hline $10 \mathrm{~min}$ & 0.55 & 0.45 & 0.75 \\
$60 \mathrm{~min}$ & 0.34 & 0.66 & 0.04 \\
$1440 \mathrm{~min}$ & 0.80 & 0.20 & 0.79 \\
\hline
\end{tabular}

for high-latitude geomagnetic variability. At least for finer scales, 10 and $60 \mathrm{~min}$, nonlinear fluctuations are strongly related to multifractal processes where the MHD turbulence can play an important role. Thus, the analysis of a standard multifractal turbulent process was shown for comparison. It was found that the multifractal and singularity spectra, for the $60 \mathrm{~min}$ characteristic time scale, is in disagreement with the standard turbulent model. In the scope of turbulent geomagnetic fluctuations, different types of singularity spectra can describe the fluctuations in direct dissipation or loading-unloading regimes of the solar windmagnetosphere interaction (Vörös, 2000). Thus, the singular behavior for low-latitude $60 \mathrm{~min}$ geomagnetic fluctuations can be interpreted as being the response of an out of equilibrium process, possibly related to the main cause of storms detected by means of the $D_{s t}$ index. Complementarily, the multifractality in our analysis also was characterized for the quiet period before the impulsive and the most intermittent phase of the event. This fact is in good agreement to the results found by Balasis et al. (2006), showing that components of the magnetospheric field can have their variabilities from internal origin.

Recently, Wanliss et al. (2005) pointed out that the ring current is always out of equilibrium and its related dynamics is an important source for multifractality found in $D_{s t}$ index time series, even for quiet or active periods. This phenomenological hypothesis may represent an opportunity to modeling geomagnetic fluctuations in a high resolution spatio-temporal framework, where the structure of the ring current as a function of the latitude can be considered. From the point of view of statistical models, non-Gaussian fluctuations can be incorporated by using the nonextensive approach (Tsallis, 1988) as in Bolzan et al. (2005b). On the other hand, Gotoh and Kraichnan (2004) have mentioned that any nonextensive representation of turbulence requires critical examination of the turbulent plasma environment. However, recent studies have shown that non-gaussian fluctuation is responsible for the presence of extreme events in space plasmas. Recently, using a non-extensive approach, Balasis et al. (2008) found out the emergence of two distinct phases: (i) the phase where the intense magnetic storms cause a higher degree of magnetic field organization, and (ii) the phase which characterizes the normal periods with lower magnetic field coherence. The phase (i) may be associated with the presence of different kinds of large scale coherent structures, also pointed out by Chang et al. (2006). Such self-organized dynamics is also observed from our results. We found that to the quiet period (nine days before the disturbance) different multifractal signatures appears when compared with the disturbed period.

In summary, an alternative theoretical framework needs to be developed, taking into account the nature of the highresolution observations of the geomagnetic field fluctuations. Arimitsu and Arimitsu (2004), have developed $f(\alpha)$ model based on a wide range of non-Gaussian fluctuations. Thus, as an extension of the technique proposed here taking into account nonextensivity and phase transition in the sense discussed by Wanliss and Dobias (2007) and Balasis et al. (2008), is currently in progress. Also new data analysis approaches more robust against spuricus statistical fluctuations can be addressed in our work (e.g. Chang and $\mathrm{Wu}$, 2008; Rosa et al., 2008).

\section{Appendix A}

In the theory of multifractal statistics the so-called p-model is a canonical mathematical system that describes energy cascade processes in turbulent flows (e.g. Rodrigues Neto et al., 2001). Then we performed the theoretical multiplicative cascade p-model as a canonical reference in our analysis. According to Halsey et al. (1986), the p-model is given by (Eqs. 2.33 and 2.31, p. 1145 from Halsey et al., 1986):

$\alpha=\frac{\log _{2} p_{1}+(w-1) \log _{2} p_{2}}{\log _{2} l_{1}+(w-1) \log _{2} l_{2}}$

and

$f(\alpha)=\frac{(w-1) \log _{2}(w-1)-w \log _{2} w}{\log _{2} l_{1}+(w-1) \log _{2} l_{2}}$

where $w$ is a free parameter and $l_{1}=l_{2}=1 / 2$ if the eddies are equal as a two-scale Cantor set. In the p-model, the largest coherent structure is assumed to be built up by a specific energy flux per unit length and then a scale-independent spaceaveraged cascade rate occurs. In this process the flux density is transferred to the two smaller eddies with the same length but different flux probabilities $p_{1}$ and $p_{2}\left(p_{1}+p_{2}=1\right)$. The process is repeated several times with $p_{1}$ and $p_{2}$ randomly distributed, being the asymmetric breakdown in the fragmentation process driven by the parameter $p=p_{1}=1-p_{2}$. The common value of $p_{1}=p_{2}=0.5$ corresponds to the homogeneous energy transfer rate with no intermittency effects. The values of $p>0.5$ corresponds to an intermittent turbulence. In the present work, we use a maximum likelihood algorithm to fit the $\mathrm{p}$-model in each characteristic scale. The derived values for the $\mathrm{p}$-model's parameters are presented in Table A1.

Acknowledgements. The authors wish to acknowledge Ronaldo Martins de Carvalho, Observatório Nacional do Rio de Janeiro, for kindly providing the Vassoura's Observatory 
Geomagnetic data. This work was supported by FAPESP (grant no. 2005/00511-9) and CNPq (grants no. 310431/2006-9 and 307931/2006-4). Thanks also to referrers for fruitful comments given by this work.

Topical Editor I. A. Daglis thanks two anonymous referees for their help in evaluating this paper.

\section{References}

Arimitsu, T. and Arimitsu, N.: Multifractal analysis of fluid particle accelerations in turbulence, Physica D, 1-4, 218-230, 2004.

Arneodo, A., Bacry, E., and Muzy, J. F.: The thermodynamics of fractals revisited with wavelets, Physica A, 213(1-2), 232-275, 1995.

Balasis, G., Daglis, I. A., Kapiris, P., Mandea, M., Vassiliadis, D., and Eftaxias, K.: From pre-storm activity to magnetic storms: a transition described in terms of fractal dynamics, Ann. Geophys., 24, 3557-3567, 2006,

http://www.ann-geophys.net/24/3557/2006/.

Balasis, G., Daglis, I. A., Papadimitriou, C., Kalimeri, M., Anastasiadis, A., and Eftaxias, K.: Dynamical complexity in $D_{s t}$ time series using non-extensive Tsallis entropy, Geophys. Res. Lett., 35, L14102, doi:10.1029/2008GL034743, 2008.

Biskamp, D. and Schwarz, E.: On two-dimensional magnetohydrodynamic turbulence, Phys. Plasmas, 8(7), 3282-3292, 2001.

Bolzan, M. J. A., Ramos, F. M., Sá, L. D. A., Rodrigues Neto, C., and Rosa, R. R.: Analysis of fully developed turbulence above and inside Amazon forest canopy by means of a generalized entropy theory, J. Geophys. Res., 107, D20, doi:10.1029/2001JD000378, 2002.

Bolzan, M. J. A., Sahai, Y., Fagundes, P. R., Rosa, R. R., Ramos, F. M., and Abalde, J. R.: Intermittency analysis of geomagnetic storm time-series observed in Brazil, J. Atmos. Solar-Terr. Phys., 67(14), 1365-1372, 2005a.

Bolzan, M. J. A., Sahai, Y., Fagundes, P. R., Rosa, R. R., Ramos, F. M., and Abalde, J. R.: Generalized thermo statistics and wavelet analysis of solar wind and proton density variability, J. Atmos. Solar-Terr. Phys., 67(14), 1365-1372, 2005b.

Bunde, A. and Havlin, S.: Fractals and Disordered Systems, 2nd ed., Springer-Verlag, Berlin, 1996.

Chapman, S. C., Watkins, N. W., Dendy, R. O., Helander, P., and Rowlands, G.: A simple avalanche model as an analogue for magnetospheric activity, Geophys. Res. Lett., 25(13), 23972400, 1998.

Chang, T.: Self-organized criticality, multi-fractal spectra, sporadic localized reconnections and intermittent turbulence in the magnetotail, Phys. Plasmas, 6, 4137-4145, 1999.

Chang, T. and Wu, C.-C.: Rank-ordered multifractal spectrum for intermittent fluctuations, Phys. Rev. E, Rapid Communications, 77, 045401(R), doi:10.1103/PhysRevE.77.045401, 2008.

Enescu, B., Ito, K., and Struzik, Z.: Wavelet-based multiscale resolution analysis of real and simulated time-series of earthquakes, Geophys. J. Int., 164(1), 63-74, 2006.

Farge, M.: Wavelet transforms and their applications to turbulence, Ann. Rev. Fluid Mech., 24, 395-457, 1992.

Frisch, U.: Turbulence, Cambridge University Press, New York, 1995.

Gotoh, T. and Kraichnan, R. H.: Turbulence and tsallis statistics, Physica D, 193(1-4), 231-244, 2004.
Halsey, T. C., Jensen, M. H., Kadanoff, L. P., Procaccia, I., and Shraiman, B. I.: Fractal measures and singularities - The characterization of strange sets, Phys. Rev. A, 33(2), 1141-1151, 1986.

Kolmogorov A. N.: The local structure of turbulence in an incompressible viscous flow for very high Reynolds numbers, C. R. Acad. Sci., SSSR, 30, 301-305, 1941 (see also in Proc. R. Soc. London A., 434, 9-13, 1991).

Kovács, P., Carbone, V., and Vörös, Z.: Wavelet-based filtering of intermittent events from geomagnetic time-series, Planet. Space Sci., 49, 1219-1231, 2001.

Kraichnan, R. H.: Lagrangian-history closure approximation for turbulence, Phys. Fluids, 8, 575-598, 1965.

Lui, A. T. Y.: Multiscale phenomena in the near-Earth magnetosphere, J. Atmos. Solar-Terr. Phys., 64, 125-143, 2002.

Mallat, S. G. and Hwang, W. L.: Singularity Detection and Processing with Wavelets, IEEE Trans. on Information Theory, 38, 617-643, 1992.

Meneveau, C. and Sreenivasan, K. R.: Simple multifractal cascade model for fully developed turbulence, Phys. Rev. Lett., 59(13), 1424-1427, 1987.

Muzy, J. F., Bacry, E., and Arneodo, A.: Wavelets and Multifractal formalism for singular signals: Application to turbulence data, Phys. Rev. Lett., 67(25), 3515-3518, 1991.

Oswiecimka, P., Kwapien, J., and Drozdz, S.: Wavelet versus detrended fluctuation analysis of multifractal structures, Phys. Rev. E, 74, 016103, doi:10.1103/PhysRevE.74.016103, 2006.

Papas, A. R. R., Barreto, L. M., and Seixas, N. A. B.: Statistical study of magnetic disturbances at the Earth's surface, J. Atmos. Solar-Terr. Phys., 68(8), 930-936, 2006.

Ramos, F. M., Bolzan, M. J. A., Sá, L. D. A., Rosa, R. R., and Rodrigues Neto, C.: Atmospheric turbulence within and above an Amazon Forest, Physica D-Nonlinear Phenomena, 193(1-4), 278-291, 2004.

Rodrigues Neto, C., Zanandrea, A., Ramos, F. M., Rosa, R. R., Bolzan, M. J. A., and Sá, L. D. A.: Multiscale analysis from turbulent time series with wavelet transform, Physica A, 295(12), 215-218, 2001.

Rosa, R. R., Karlicky, M., Veronese, T. B., Vijaykumar, N. L., Sawant, H. S., Borgazzi, A. I., Dantas, M. S., Barbosa, E. B. M., Sych, R. A., and Mendes, O.: Gradient pattern analysis of short solar radio bursts, Adv. Space Res., 42(5), 844-851, 2008.

Stanley, H. E. and Meakin, P.: Multifractal phenomena in physics and chemistry, Nature, 335, 405-409, 1988.

Struzik, Z. R.: Determining local singularity strengths and their spectra with the wavelet transform, Fractals, 8, 163-179, 2000.

Tsallis, C.: Possible generalization of Boltzmann-Gibbs statistics, J. Stat. Phys., 58, 1-2, 1988.

Vicsek, T.: Fractal Growth Phenomena, 2nd ed., World Scientific, Singapore, 1993.

Vörös, Z.: On multifractality of high-latitude geomagnetic fluctuations, Ann. Geophys., 18, 1273-1282, 2000, http://www.ann-geophys.net/18/1273/2000/.

Vörös, Z., Jankovičová, D., and Kovács, P.: Scaling and singularity characteristics of solar wind and magnetospheric fluctuations, Nonlin. Processes Geophys., 9, 149-162, 2002, http://www.nonlin-processes-geophys.net/9/149/2002/.

Wanliss, J. A., Anh, V. V., Yu, Z.-G., and Watson, S.: Multifractal modeling of magnetic storms via symbolic dynamics analysis, J. Geophys. Res., 110, A08214, doi:10.1029/2004JA010996, 2005. 
Wanliss, J. A. and Dobias, P.: Space storm as a phase transition, J. Atmos. Solar-Terr. Phys., 69, 675-684, 2007.

Weng, H. Y. and Lau, K. M.: Wavelets, period-doubling, and timefrequency localization with application to organization of convection over the tropical western pacific, J. Atmos. Sci., 51(17), 2523-2541, 1994.
Weygand, J. M., Kivelson, M. G., Khurana, K. K., Schwarzl, H. K., Thompson, S. M., McPherron, R. L., Balogh, A., Kistler, L. M., Goldstein, M. L., Borovsky, J., and Roberts, D. A.: Plasma sheet turbulence observed by CLUSTER II, J. Geophys. Res., 110, A01205, doi:10.1029/2004JA010581, 2005. 